\title{
Nitrate tolerance, oxidative stress, and mitochondrial function: another worrisome chapter on the effects of organic nitrates
}

\author{
John D. Parker \\ Division of Cardiology, Mount Sinai and University Health Network Hospitals, and \\ Department of Medicine, University of Toronto, Toronto, Ontario, Canada
}

\begin{abstract}
A shortcoming in the clinical use of organic nitrates is the development of tolerance. Recent data have suggested that the denitrification of organic nitrates is mediated by mitochondrial aldehyde dehydrogenase and that dysfunction of this enzyme is an important cause of tolerance. In this issue of the JCI, evidence in support of this hypothesis is presented in an in vivo model of nitrate tolerance (see the related article beginning on page 482).
\end{abstract}

J. Clin. Invest. 113:352-354 (2004). doi:10.1172/JCI200421003.

Organic nitrates have been used widely in the treatment of both coronary artery disease and congestive heart failure. The most commonly used organic nitrates are nitroglycerin, isosorbide dinitrate, and isosorbide-5-mononitrate (1). The organic nitrates are prodrugs, undergoing a biotransformation process that is generally believed to yield NO or some NO adjunct as their vasoactive metabolite. Despite clear benefits when given acutely, the organic nitrates rapidly lose their hemodynamic and clinical effectiveness when used as sustained therapy, a phenomenon known as nitrate tolerance. The mechanism of nitrate tolerance has been the subject of numerous investigations and much debate. Initially, it was hypothesized that tolerance was caused by abnormalities in the nitrate biotransformation process (termed mechanism-based or classical tolerance); however, those studies were limited by our lack of understanding of the biochemical processes involved. Many other theories have been proposed, including reflex hormonal and neurochemical mechanisms (termed pseudotolerance), although a clear

Address correspondence to: John D. Parker, Mount Sinai Hospital, Suite 1609,

600 University Avenue, Toronto, Ontario M5G 1X5, Canada. Phone: (416) 586-4794; Fax: (416) 586-8413; E-mail: jdp@ca.inter.net. Conflict of interest: The author has declared that no conflict of interest exists.

Nonstandard abbreviation used: mito-

chondrial aldehyde dehydrogenase (ALDH-2). cause-and-effect relationship could not be established $(2,3)$.

\section{The free radical hypothesis of nitrate tolerance}

In 1995 Münzel and colleagues described that 3 days of nitroglycerin treatment in rabbits was associated with increased superoxide production by the aortic endothelium (4). They demonstrated that tolerance to nitroglycerin was dependent on the endothelium and went on to hypothesize that the increased free radical production, by scavenging $\mathrm{NO}$ released by the nitrate, was the mechanism underlying the development of tolerance. This observation, termed the free radical hypothesis of nitrate tolerance, led to a series of observations examining the mechanism and enzymatic sources of increased free radical production during nitrate therapy, the details of which have recently been reviewed $(2,3)$. The observation that therapy with organic nitrates increased vascular free radical production provided a coherent explanation for many previous observations concerning nitrate tolerance. Support for the relevance of these observations came with a number of studies suggesting that nitrate tolerance could be modified with the concurrent administration of antioxidant compounds (5). Although the hypothesis that excess free radical generation reduced the bioavailability of NO produced by the nitrates was appealing, studies contin- ued to document abnormalities in the biotransformation process, suggesting that abnormalities in the denitrification process were also involved (6).

\section{The biotransformation} of nitroglycerin

In 2002, the laboratory of Jonathan Stamler reported that mitochondrial aldehyde dehydrogenase (ALDH-2) played a primary role in the biotransformation of nitroglycerin (7). In this report, they documented the importance of this enzyme for the liberation of NO from nitroglycerin. They demonstrated that nitrate tolerance is associated with evidence of dysfunction of ALDH-2 with subsequent loss of nitroglycerin effects. In this issue of the JCI, Sydow and colleagues, from the laboratory of Thomas Münzel, provide important additional data concerning the role of this enzyme system in an in vivo model of nitrate tolerance (8). They provide evidence that sustained treatment with nitroglycerin in vivo is associated with reduced nitroglycerin biotransformation by ALDH-2 and that this abnormality in nitrate biotransformation is associated with increased mitochondrial formation of free radical oxygen species (Figure 1). Their findings are supportive of the initial observations of Chen et al. (7) and provide important new data. Chen et al. made use of an in vitro model of nitrate tolerance with relatively shortterm exposure to high concentrations of nitroglycerin. In the present paper by Sydow et al., the importance of ALDH-2 and impairment of biotransformation is confirmed in an in vivo model of sustained nitroglycerin therapy. More importantly, this manuscript links the problem of abnormalities in nitrate biotransformation with increased free radical bioavailability during sustained nitrate therapy. They document that in vivo nitrate exposure is associated with an increase in free radical production by cardiac mitochondria. The importance of mitochondria in the metabolism of nitroglycerin is emphasized by the observation that cultured endothelial cells rendered devoid of mitochondrial function displayed a marked decrease in nitroglycerin-induced production of cyclic GMP. 


\section{Continued controversy}

The present findings by Sydow et al. (8) link the free radical hypothesis of nitrate tolerance with the original concept that nitrate tolerance was associated with abnormalities in nitrate biotransformation. Despite the clarity of the observations by Chen et al. (7) and the present work (8), many questions remain unanswered. Not all data are in agreement with the conclusion that ALDH-2 is a major mechanism of nitrate biotransformation. Difabio et al., using a similar in vivo model of nitrate tolerance, found that inhibitors of ALDH-2 caused a similar shift in the relaxation responses of tolerant and nontolerant aortae (9). Those authors concluded that mitochondrial biotransformation of nitroglycerin plays only a minor role in the overall vascular metabolism of nitroglycerin. These contradictory results highlight the potential effect that different experimental conditions can have on observations of nitrate effects and tolerance. Another intriguing question relates to the tissue distribution of this nitrate esterase. Studies to date have readily isolated it from vascular, hepatic, and cardiac homogenates. It is surprising that nitrate therapy could have important metabolic effects in such a wide range of tissues, as more than a century of clinical experience suggests that the pharmacodynamic effects of organic nitrates are much more limited. The finding that the nitrates undergo biotransformation and release $\mathrm{NO}$ in such a wide distribution of tissues is sur- prising and raises important questions concerning the clinical applicability of this model of nitrate effects.

The nature of the initial trigger leading to the increase in free radical production observed during nitrate therapy remains unknown. To date, multiple enzymatic sources have been described, but the primary mechanism has not been identified. In the present publication by Sydow et al. (8), the authors state that nitroglycerin, superoxide, and peroxynitrite are all capable of directly inhibiting ALDH-2. A potential hypothesis is that the biotransformation process itself causes the initial increase in free radical production that then promotes a cascade of secondary increases in free radical production and/or bioavailability. Continued

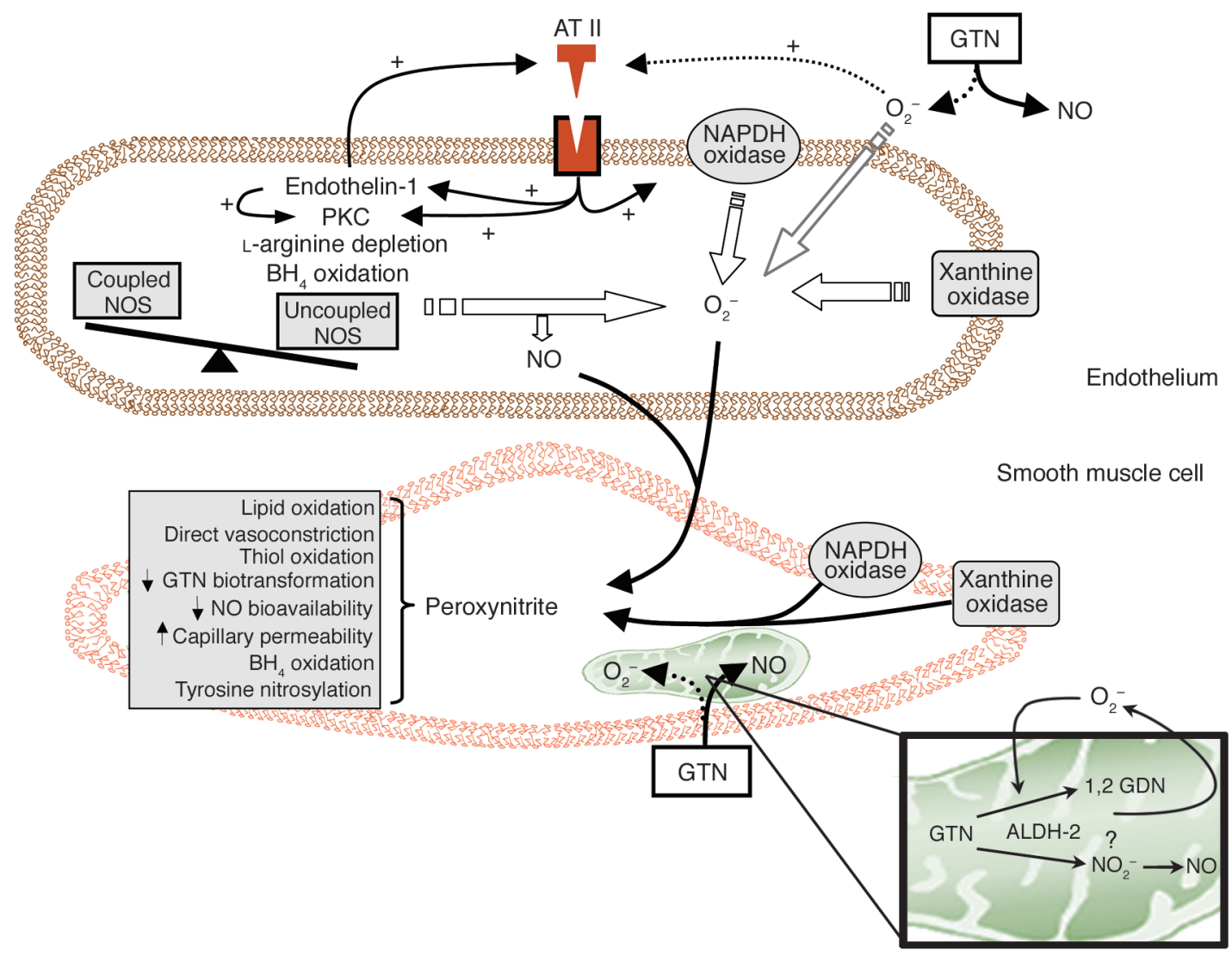

Figure 1

Diagram depicting the proposed pathways for the development of nitrate tolerance. Both endothelial and smooth muscle cells are involved in these processes. Superoxide anion is produced by membrane oxidases and, possibly, during nitroglycerin (glyceryl trinitrate; GTN) biotransformation (dashed arrow). Increased angiotensin II (AT II) production and responsiveness and increased oxidative stress may result in uncoupling of nitric oxide synthase (NOS) and further production of reactive oxygen species and peroxynitrite. In turn, these oxidant free radicals may cause many of the abnormalities observed in nitrate tolerance. Isoprostane formation and increased capillary permeability might be responsible for plasma volume expansion, and tyrosine nitrosylation and thiol group oxidation might be responsible for inactivation of multiple enzymes, including those involved in the biotransformation of nitroglycerin. Tetrahydrobiopterin $\left(\mathrm{BH}_{4}\right)$ oxidation may cause NOS uncoupling. Figure modified with permission from Circulation (2). PKC, protein kinase $\mathrm{C} ; \mathrm{O}_{2}{ }^{-}$, superoxide anion; GDN, glyceryl dinitrate; $\mathrm{NO}_{2}^{-}$, nitrite. 
improvements in our understanding of the exact site and mechanism of the biotransformation of organic nitrates, including a better understanding of the biochemical intermediaries involved, may well lead to a better understanding of the cause of this phenomena.

\section{Sustained therapy with organic nitrates: is it safe?}

The concept that sustained nitrate therapy causes increased production of free radicals has a number of important implications. Although their clinical efficacy during chronic therapy had been questioned for many years because of phenomena of tolerance, it had generally been assumed that the organic nitrates were safe drugs with no potential for long-term toxicity. Some authors proposed that organic nitrates, as exogenous NO donors, would be beneficial in the setting of chronic atherosclerosis, as they could compensate for the decrease in NO bioavailability that characterizes that disease state. Although the hypothesis was raised, it has never been tested in a long-term trial of clinical outcomes.
Today, multiple lines of evidence suggest that this assumption of safety is likely to be incorrect. A series of human observations have documented that therapy with nitroglycerin causes severe abnormalities in endothelial function, as defined by abnormalities in the response to endothelium-dependent vasodilators, in both the coronary and peripheral arterial circulation (reviewed in refs. 2 and 3 ). The present study (8), remarkably, documents that therapy with nitroglycerin causes increased free radical production by cardiac mitochondria as well as abnormalities in aortic ALDH-2 activity. These observations suggest that therapy with nitroglycerin can cause important abnormalities in mitochondrial respiration and oxygen consumption. Although the observation that this occurs in cardiac mitochondria is somewhat surprising, it again raises questions concerning the safety of these agents in the long-term therapy of cardiac disease. Taken together, these observations suggest that the time has come to mount an appropriately powered clinical trial examining the efficacy and safety of sus- tained therapy with organic nitrates for those diseases for which they are commonly used.

1. Parker, J.D., and Parker, J.O. 1998. Nitrate therapy for stable angina pectoris. N. Engl. J. Med. 338:520-531.

2. Gori, T., and Parker, J.D. 2002. The puzzle of nitrate tolerance: pieces smaller than we thought? Circulation. 106:2404-2408.

3. Gori, T., and Parker, J.D. 2002. Nitrate tolerance: a unifying hypothesis. Circulation. 106:2510-2513.

4. Munzel, T., Sayegh, H., Freeman, B.A., Tarpey, M.M., and Harrison, D.G. 1995. Evidence for enhanced vascular superoxide anion production in nitrate tolerance. A novel mechanism underlying tolerance and cross-tolerance. J. Clin. Invest. 95:187-194

5. Bassenge, E., Fink, N., Skatchkov, M., and Fink, B. 1998. Dietary supplement with vitamin C prevents nitrate tolerance. J. Clin. Invest. 102:67-71.

6. Sage, P.R., et al. 2000. Nitroglycerin tolerance in human vessels: evidence for impaired nitroglyc erin bioconversion. Circulation. 102:2810-2815.

7. Chen, Z., Zhang, J., and Stamler, J.S. 2002. Identification of the enzymatic mechanism of nitroglycerin bioactivation. Proc. Natl. Acad. Sci. U. S. A. 99:8306-8311.

8. Sydow, K., et al. 2004. Central role of mitochondrial aldehyde dehydrogenase and reactive oxygen species in nitroglycerin tolerance and cross-tolerance. J. Clin. Invest. 113:482-489. doi:10.1172/JCI200419267

9. DiFabio, J., Ji, Y., Vasiliou, V., Thatcher, G.R., and Bennett, B.M. 2003. Role of mitochondrial aldehyde dehydrogenase in nitrate tolerance. Mol. Pharmacol. 64:1109-1116.

\section{Tandem mass spectrometry in discovery of disorders of the metabolome}

\section{Arnold W. Strauss}

Department of Pediatrics, Vanderbilt Children's Hospital, Vanderbilt University, Nashville, Tennessee, USA

Genetic disorders of amino acid and fatty acid metabolism can be detected with tandem mass spectrometry (MS/MS). MS/MS screening of mice subjected to chemical mutagenesis (see the related article beginning on page 434) defined a new disorder of branched-chain amino acid metabolism resembling human maple syrup urine disease. This approach has general application to the discovery of gene function in developmental and metabolic disorders.

J. Clin. Invest. 113:354-356 (2004). doi:10.1172/JCI200420924.
The post-genomic era has arrived and has created an exciting environment for the discovery of genetic disorders, multigenic etiologies of disease, and pharmacogenomics, and the delineation of gene function, with unusual approaches (1). In this issue of the JCI, Y.-T. Chen, an accomplished biochemical geneticist, and coworkers use an intriguing process with potential general application to mammalian disease and congenital anomalies to demonstrate the phenotype of a new metabolic disorder (Figure 1). The results document the usefulness of studying the "metabolome," a term that refers to the low-molecular-weight compounds present in organisms that are substrates, products, and regulatory molecules in macromolecular metabolic pathways. This commentary projects three major conclusions. First, screening of mutagenized animals with clinically relevant approaches will define phenotypes similar to critical human disorders. Second, the study of the metabolome will likely define critical compounds, relevant genetic disorders, and modifying genes in animal models that will be revealing in our understanding of human disease. Third, highly sensitive technologies applied to both animal models and humans will result in the discovery of new disorders; the aberrant, causative genes; and new treatments.

\section{Metabolomics, screening, and tandem mass spectrometry}

Following chemical mutagenesis of mice using $N$-ethyl- $N$-nitrosourea and 